\title{
Solving Benjamin-Bona-Mahony equation by using the sn-ns method and the tanh-coth method
}

\author{
HAMİ GÜNDOĞDU AND ÖMER FARUK GÖZÜKIZIL
}

\begin{abstract}
In this study, we consider the Benjamin Bona Mahony equation which is in the form of $u_{t}+u_{x}+u u_{x}-u_{x x t}=0$. The sn-ns method and the tanh-coth method have been applied to this equation. And then, exact solutions have been obtained.
\end{abstract}

\section{INTRODUCTION}

Nonlinear phenomena generally occurs in most fields of modern sciences and engineering. Most of the encountered problems in these areas are modeled by nonlinear partial differentail equations (NLPDEs shortly). Therefore, finding solutions to NLPDEs has an important role in nonlinear science problems. In this respect, a great deal of research has been made in order to handle NLPDEs during the past decades. And then, many methods have been established and used for obtaining exact solutions to NPLDEs. Some of the powerful methods are the generalized tanh method [1], the tanh-coth method (extended tanh method) [2], the tanh-sech method [3, 4], sine-cosine method [5]-[6], the exp-function method [7], the projective Ricatti equations method [8], the generalized projective Ricatti equations method [9], the (G'/G)-expansion method [10], and the sn-ns method[11].

This work is related to generalized Benjamin Bona Mahony equation (BBM) given by

$$
u^{n} u_{x}-u_{x x t}+u_{t}-\alpha u_{x x}+u_{x}=0, \quad 0 \leq \alpha \leq 1, \quad n \in N
$$

where $u(x, t)$ is the solution of equation (1) and considered as a class of non-periodic functions defined on $-\infty \leq x \leq \infty$ and $t \geq 0$.

In our work, the case of $\alpha=0$ and $n=1$ has been investigated and the equation (1) has taken the following form

$$
u_{t}+u_{x}+u u_{x}-u_{x x t}=0 .
$$

2000 Mathematics Subject Classification. Primary: 35C07; Secondary: 35C09, 35C09.

Key words and phrases. Benjamin Bona Mahony equation (BBM), the sn-ns method, tanh-coth method, elliptic function solution, trigonometric solution. 
The equation (2) is called Benjamin Bona Mahony equation (BBM). It is also known as the regularized long-wave equation. For the first time, it was introduced in 1970s by Benjamin et al. [12] as an alternative to the well-known Korteweg de Vries (KdV) equation [13] given by

$$
u_{t}+u_{x}+u_{x x x}+u u_{x}=0 .
$$

The KdV equation has been used for modelling water waves of small amplitude and large wavelength. For more detail about BBM equation, it is referred to [14]-[17] and the references therein.

This work is arranged as follows. Firstly, we have mentioned about the methods in Section 2. After that, we have applied these methods to equation (2) in Section 3. Then, we have introduced new traveling wave solutions in Section 4. Finally, some conclusions have been given.

\section{Outline of the Methods}

In this section, summary of the methods has been given.

2.1. The tanh-coth method. Wazwaz has given the tanh method in [2] as in the following manner.

First, let us consider a general form of the nonlinear partial differential equation (NLPDE)

$$
P\left(u, u_{t}, u_{x}, u_{x x}, u_{x t}, u_{t t}, u_{x x t}, \ldots\right)=0 .
$$

To obtain the traveling wave solutions of equation (4), the wave variable, $\xi=x-\mathrm{V} t$, is introduced so that $u(x, t)=U(\mu \xi)$.

Now we can give the partial derivatives of $u(x, t)$ in the form of ordinary derivatives of $U(\mu \xi)$ as follows:

$$
\begin{aligned}
\frac{\partial u}{\partial t} & =-V \mu \frac{\mathrm{d} U}{\mathrm{~d} \xi}, \\
\frac{\partial u}{\partial x} & =\mu \frac{\mathrm{d} U}{\mathrm{~d} \xi}, \\
\frac{\partial u^{2}}{\partial x^{2}} & =\mu \frac{\mathrm{d}^{2} U}{\mathrm{~d} \xi^{2}},
\end{aligned}
$$

and so on for higher derivatives.

After this step, the equation (4) is converted into the following ordinary differential equation (ODE)

$$
Q\left(U, U^{\prime}, U^{\prime \prime}, \ldots\right)=0 \text {. }
$$

Then, if each terms of the resulting ODE contains derivatives with respect to $\xi$, by integrating this equation and by considering the constant of integration to be zero, we get a simplified ODE as in (5).

Here we introduce a new independent variable

$$
Y=\tanh (\mu \xi)
$$


so that it leads to the change of derivatives as in the followings:

$$
\begin{aligned}
\frac{\mathrm{d}}{\mathrm{d} \xi}= & \mu\left(1-Y^{2}\right) \frac{\mathrm{d}}{\mathrm{d} Y}, \\
\frac{\mathrm{d}^{2}}{\mathrm{~d} \xi^{2}}= & \mu^{2}\left(1-Y^{2}\right) \frac{\mathrm{d}^{2}}{\mathrm{~d} Y^{2}}-2 \mu^{2} Y\left(1-Y^{2}\right) \frac{\mathrm{d}}{\mathrm{d} Y}, \\
\frac{\mathrm{d}^{3}}{\mathrm{~d} \xi^{3}}= & \mu^{3}\left(1-Y^{2}\right)^{3} \frac{\mathrm{d}^{3}}{\mathrm{~d} Y^{3}}-6 \mu^{3} Y\left(1-Y^{2}\right)^{2} \frac{\mathrm{d}^{2}}{\mathrm{~d} Y^{2}} \\
& +2 \mu^{3}\left(1-Y^{2}\right)\left(3 Y^{2}-1\right) \frac{\mathrm{d}}{\mathrm{d} Y} .
\end{aligned}
$$

where other derivatives can be derived in a similar way. Now the approach of the form

$$
U(\mu \xi)=S(Y)=\sum_{k=0}^{M} a_{k} Y^{k}+\sum_{k=0}^{M} b_{k} Y^{-k}
$$

is introduced, where $M$ is usually a positive integer that we must determine. Putting (7) into the equation (5) gives an equation in power of $Y$. To obtain the parameter $M$, the linear terms of highest order in the resulting equation with the highest-order nonlinear terms are equalized. After $M$ is determined, all the coefficients of same power of $Y$ in the resulting equation are collected respectively. Then, these coefficients have to vanish.

This will give us a system of algebraic equations involving the $a_{k}$ and $b_{k}$, $(k=0, \ldots, M), \mathrm{V}$ and $\mu$.

Now, these $a_{k}, b_{k}, V$ and $\mu$ should be determined by solving the algebraic system. After that, putting these parameters into (7) provide us with analytic solutions in a closed form.

2.2. The sn-ns method. This method has been introduced by H. S. Alvaro [11] in detail. With the aid of this method, we have searched for the traveling wave solutions to nonlinear partial differential equation of the form

$$
P\left(u, u_{t}, u_{x}, u_{x x}, u_{x t}, u_{t t}, u_{x x t}, \ldots\right)=0 .
$$

Let us consider the wave transformation

$$
u(x, t)=v(\theta(\xi)), \xi=x+\lambda t+\xi_{0}
$$

where $\lambda$ is a constant and $\xi_{0}$ is an arbitrary constant. And, $\theta=\theta(\xi)$ is a suitable function that makes the transformation simple. For this purpose, we take $\theta(\xi)$ as the identity function, i.e. $\theta(\xi)=\xi$.

Using the transformation (9) and putting ordinary derivative of $v(\xi)$ instead of the partial derivatives of $u(x, t),(8)$ converts to an ordinary differential equation (ODE) with respect to the function $v(\xi)$

$$
Q\left(v, v^{\prime}, v^{\prime \prime}, \ldots\right)=0
$$

with $Q$ being a polynomial with respect to variables $v, v^{\prime}, v^{\prime \prime}, \ldots$ 
In this method, we seek the traveling wave solutions to (10) in the form

$$
v(\xi)=a_{0}+\sum_{j=1}^{n} a_{j} \operatorname{sn}^{j}(k \xi \mid m)+\sum_{j=1}^{n} b_{j} \mathrm{~ns}^{j}(k \xi \mid m) .
$$

To determine the coefficients $\left(a_{0}, a_{j}, b_{j}\right)$, the balancing constant $(n), \lambda$ and $k$, the same process above can be followed. Generally, we find $n=1,2$ and then $v(\xi)$ takes the forms

$$
v(\xi)=a_{0}+a_{1} \operatorname{sn}(k \xi \mid m)+b_{1} \operatorname{ns}(k \xi \mid m),
$$

$$
\left.v(\xi)=a_{0}+a_{1} \mathrm{sn}(k \xi \mid m)\right)+b_{1} \mathrm{~ns}(k \xi \mid m)+a_{2} \mathrm{sn}^{2}(k \xi \mid m)+b_{2} \mathrm{~ns}^{2}(k \xi \mid m) .
$$

For some special values of $m$, the following equalities are yielded.

For $m=1$,

$$
\operatorname{sn}(k \xi, 1)=\tanh (k \xi) \text { and } \operatorname{ns}(k \xi, 1)=\operatorname{coth}(k \xi),
$$

and for $m=0$,

$$
\operatorname{sn}(k \xi, 0)=\sin (k \xi) \text { and } \operatorname{ns}(k \xi, 0)=\csc (k \xi) .
$$

Throughout the work, Mathematica has been used to deal with the tedious algebraic operations.

\section{Application of the Methods}

In this part, the methods mentioned above has been applied to the BenjaminBona-Mahony (BBM) equation given in (2).

3.1. Solving BBM equation by tanh-coth method. Using the wave variable $\xi=x-\mathrm{Vt}$ carries (2) into the ODE

$$
\mathrm{V} v^{\prime \prime}+\frac{v^{2}}{2}-v \mathrm{~V}+v=0
$$

To determine balancing constant $M$, we use the transformation $v=\phi^{M}$, $v^{\prime}=M \phi^{M+1}, v^{\prime \prime}=M(M+1) \phi^{M+2}, \ldots$ and $v^{2}=\phi^{2 M}, \ldots, v^{k}=\phi^{k M}$. Putting these into the equation (14) and balancing the power of $v^{2}$ with $v^{\prime \prime}$ gives $M=2$. So, the tanh method admits the use of the finite expansion

$$
U(\mu \xi)=S(Y)=\sum_{k=0}^{2} a_{k} Y^{k}+\sum_{k=0}^{2} b_{k} Y^{-k}
$$

where $Y=\tanh (\mu \xi)$. Substituting (15) into (14), collecting the coefficients of same powers of $Y$, and setting it equal to zero, we have found the system of equations:

$$
\begin{aligned}
& Y^{8}: 12 a_{2} \mathrm{~V} \mu^{2}+a_{2}^{2}=0 \\
& Y^{7}: 4 a_{1} \mathrm{~V} \mu^{2}+2 a_{1} a_{2}=0, \\
& Y^{6}:-16 a_{2} \mathrm{~V} \mu^{2}+a_{1}^{2}+2 a_{0} a_{2}+2 a_{2}-2 \mathrm{~V} a_{2}=0,
\end{aligned}
$$




$$
\begin{aligned}
Y^{5}: & 2 a_{2} b_{1}-4 a_{1} \mathrm{~V} \mu^{2}+2 a_{0} a_{1}+2 a_{1}-2 \mathrm{~V}_{1}=0, \\
Y^{4}: & 2 a_{1} b_{1}+2 a_{2} b_{2}+4 a_{2} \mathrm{~V} \mu^{2}+a_{0}^{2}+2 a_{0}+4 b_{2} \mathrm{~V} \mu^{2}-2 \mathrm{~V} a_{0}=0, \\
Y^{3}: & 2 a_{0} b_{1}+2 a_{1} b_{2}+2 b_{1}-4 \mu^{2} \mathrm{~V} b_{1}-2 \mathrm{~V} b_{1}=0, \\
Y^{2} & : 2 a_{0} b_{2}+b_{1}^{2}+2 b_{2}-16 \mu^{2} \mathrm{~V} b_{2}-2 \mathrm{~V} b_{2}=0, \\
Y^{1}: & 2 b_{1} b_{2}+4 \mu^{2} \mathrm{~V} b_{1}=0, \\
Y^{0} & : 12 b_{2} \mathrm{~V} \mu^{2}+b_{2}^{2}=0 .
\end{aligned}
$$

Use of Mathematica gives the coefficients, $\mathrm{V}$ and $\mu$ as follow:

(i) $a_{0}=\frac{1}{2}, a_{1}=0, b_{1}=0, a_{2}=-\frac{3}{2}, b_{2}=0, \mathrm{~V}=\frac{1}{2}, \mu= \pm \frac{1}{2}$,

(ii) $a_{0}=\frac{1}{2}, a_{1}=0, b_{1}=0, a_{2}=0, b_{2}=-\frac{3}{2}, \mathrm{~V}=\frac{1}{2}, \mu= \pm \frac{1}{2}$,

(iii) $a_{0}=-\frac{1}{4}, a_{1}=0, b_{1}=0, a_{2}=-\frac{3}{8}, b_{2}=-\frac{3}{8}, \mathrm{~V}=\frac{1}{2}, \mu= \pm \frac{1}{4}$.

Putting these into (15) gives the following hyperbolic solutions:

$$
\begin{aligned}
& u_{1}(x, t)=\frac{1}{2}-\frac{3}{2} \tanh ^{2}\left(\frac{1}{2}\left(x-\frac{t}{2}\right)\right), \\
& u_{2}(x, t)=\frac{1}{2}-\frac{3}{2} \operatorname{coth}^{2}\left(\frac{1}{2}\left(x-\frac{t}{2}\right)\right), \\
& u_{3}(x, t)=-\frac{1}{4}-\frac{3}{8} \tanh ^{2}\left(\frac{1}{4}\left(x-\frac{t}{2}\right)\right)-\frac{3}{8} \operatorname{coth}^{2}\left(\frac{1}{4}\left(x-\frac{t}{2}\right)\right) .
\end{aligned}
$$

3.2. Solving BBM equation by sn-ns method. Let us consider the $\mathrm{BBM}$ equation given in (2). After using the wave transformation, it converts to the following ordinary differential equation w.r.t $\xi$.

$$
\mathrm{V} v^{\prime \prime}+\frac{1}{2} v^{2}-v \mathrm{~V}+v=0 .
$$

This ODE can be written as follows:

$$
v^{\prime \prime}=\left(-\frac{1}{2 \mathrm{~V}}\right) v^{2}+\left(\frac{\mathrm{V}-1}{\mathrm{~V}}\right) v
$$

which is in the form of Quadratic Duffing Equation.

Remark 3.1. In [11], Quadratic Duffing Equation is given in the form of

$$
v^{\prime \prime}(\xi)=p v^{2}(\xi)+q v(\xi)+r .
$$

Here, we have $p=-\frac{1}{2 \mathrm{~V}}, q=\left(\frac{\mathrm{V}-1}{\mathrm{~V}}\right)$ and $r=0$.

In the same way above, we can find the balancing constant $n=2$. We seek solutions to (2) in the form (13), that is

$$
\left.v(\xi)=a_{0}+a_{1} \operatorname{sn}(k \xi \mid m)\right)+b_{1} \mathrm{~ns}(k \xi \mid m)+a_{2} \mathrm{sn}^{2}(k \xi \mid m)+b_{2} \mathrm{~ns}^{2}(k \xi \mid m) .
$$

Inserting this into the equation (2) and setting all coefficients to be zero gives the following algebraic system:

$$
\begin{aligned}
& a_{1} \mu^{2} m^{2}-a_{1} a_{2}\left(\frac{-1}{2 \mathrm{~V}}\right)=0 \\
& 6 a_{2} \mu^{2} m^{2}-a_{2}^{2}\left(\frac{\mathrm{V}-1}{\mathrm{~V}}\right)=0 \\
& 4 a_{2} \mu^{2}+4 a_{2} \mu^{2} m^{2}+a_{2}\left(\frac{\mathrm{V}-1}{\mathrm{~V}}\right)-a_{1}^{2}-2 a_{0} a_{2}\left(\frac{-1}{2 \mathrm{~V}}\right)=0 \\
& 2\left(a_{2} b_{1}+a_{0} a_{1}\right)\left(\frac{-1}{2 \mathrm{~V}}\right)+a_{1} \mu^{2}+a_{1} \mu^{2} m^{2}+a_{1}\left(\frac{\mathrm{V}-1}{\mathrm{~V}}\right)=0 \\
& b_{1} \mu^{2}-b_{1} b_{2}\left(\frac{-1}{2 \mathrm{~V}}\right)=0
\end{aligned}
$$




$$
\begin{aligned}
& 6 b_{2} \mu^{2}-b_{2}^{2}\left(\frac{-1}{2 \mathrm{~V}}\right)=0 \\
& -\left(2 a_{0} b_{2}+b_{1}^{2}\right)\left(\frac{-1}{2 \mathrm{~V}}\right)+4 b_{2} \mu^{2}+4 b_{2} \mu^{2} m^{2}+b_{2}\left(\frac{\mathrm{V}-1}{\mathrm{~V}}\right)=0, \\
& 2\left(a_{0} b_{1}+a_{1} b_{2}\right)\left(\frac{-1}{2 \mathrm{~V}}\right)+b_{1} \mu^{2}+b_{1} \mu^{2} m^{2}+b_{1}\left(\frac{\mathrm{V}-1}{\mathrm{~V}}\right)=0, \\
& -\left(2 a_{1} b_{1}+2 a_{2} b_{2}+a_{0}^{2}\right)\left(\frac{-1}{2 \mathrm{~V}}\right)+2 a_{2} \mu^{2}-a_{0}\left(\frac{\mathrm{V}-1}{\mathrm{~V}}\right)+2 b_{2} \mu^{2} m^{2}=0 .
\end{aligned}
$$

Solving this system, we obtain the coefficients:

$$
\begin{aligned}
\text { (i) } a_{0} & =\frac{1}{2}\left(\frac{4 m^{2}}{\sqrt{16 m^{4}-16 m^{2}+16}}+\frac{4}{\sqrt{16 m^{4}-16 m^{2}+16}}-1\right) \\
a_{1} & =0, b_{1}=0, a_{2}=-\frac{3 m^{2}}{2 \sqrt{m^{4}-m^{2}+1}}, b_{2}=0, v=\frac{1}{2} \\
\mu & = \pm \frac{1}{\sqrt[4]{16 m^{4}-16 m^{2}+16}}, \\
\text { (ii) } a_{0} & =\frac{1}{2}\left(\frac{4 m^{2}}{\sqrt{16 m^{4}-16 m^{2}+16}}+\frac{4}{\sqrt{16 m^{4}-16 m^{2}+16}}-1\right) \\
a_{1} & =0, b_{1}=0, a_{2}=0, b_{2}=-\frac{3}{2 \sqrt{m^{4}-m^{2}+1}}, v=\frac{1}{2}, \\
\mu & = \pm \frac{1}{\sqrt[4]{16 m^{4}-16 m^{2}+16}}, \frac{4}{2 m^{2}} \\
\text { (iii) } a_{0} & =\frac{1}{2}\left(\frac{1}{\sqrt{16 m^{4}+224 m^{2}+16}}+\frac{3 m^{2}}{\sqrt{16 m^{4}+224 m^{2}+16}}-1\right) \\
a_{1} & =0, b_{1}=0, a_{2}=-\frac{1}{2 \sqrt{m^{4}+14 m^{2}+1}}, b_{2}=-\frac{1}{2 \sqrt{m^{4}+14 m^{2}+1}} \\
v & =\frac{1}{2}, \mu= \pm \frac{4}{16 m^{4}+224 m^{2}+16},
\end{aligned}
$$

(iv) $a_{0}=\frac{1}{2}\left(-\frac{m^{2}}{\sqrt{m^{4}-m^{2}+1}}-\frac{1}{\sqrt{m^{4}-m^{2}+1}}-1\right)$,

$$
\begin{aligned}
& a_{1}=0, b_{1}=0, a_{2}=\frac{6 m^{2}}{\sqrt{16 m^{4}-16 m^{2}+16}}, b_{2}=0, v=\frac{1}{2}, \\
& \mu= \pm \frac{i}{\sqrt[4]{16 m^{4}-16 m^{2}+16}},
\end{aligned}
$$

(v) $a_{0}=\frac{1}{2}\left(-\frac{m^{2}}{\sqrt{m^{4}-m^{2}+1}}-\frac{1}{\sqrt{m^{4}-m^{2}+1}}-1\right)$,

$$
\begin{aligned}
& a_{1}=0, b_{1}=0, a_{2}=0, b_{2}=\frac{6}{\sqrt{16 m^{4}-16 m^{2}+16}}, v=\frac{1}{2}, \\
& \mu= \pm \frac{i}{\sqrt[4]{16 m^{4}-16 m^{2}+16}},
\end{aligned}
$$




$$
\text { (vi) } \begin{aligned}
a_{0} & =\frac{1}{2}\left(-\frac{m^{2}}{\sqrt{m^{4}+14 m^{2}+1}}-\frac{1}{\sqrt{m^{4}+14 m^{2}+1}}-1\right) \\
a_{1} & =0, b_{1}=0, a_{2}=\frac{6 m^{2}}{\sqrt{16 m^{4}+224 m^{2}+16}}, \\
b_{2} & =\frac{6}{\sqrt{16 m^{4}+224 m^{2}+16}}, v=\frac{1}{2}, \mu= \pm \frac{i}{\sqrt[4]{16 m^{4}+224 m^{2}+16}} .
\end{aligned}
$$

Putting these into (13), we obtain the Jacobi elliptic function solutions as follows:

$$
\begin{aligned}
& \left.v_{1}(\xi)=\frac{1}{2}\left(-1+\frac{4}{\sqrt{16 m^{4}-16 m^{2}+16}}\right)+\frac{4 m^{2}}{\sqrt{16 m^{4}-16 m^{2}+16}}\right)- \\
& \frac{3 m^{2}}{2 \sqrt{m^{4}-m^{2}+1}} \operatorname{sn}^{2}\left(\frac{1}{\sqrt[4]{16 m^{4}-16 m^{2}+16}} \xi \mid m\right), \\
& \left.v_{2}(\xi)=\frac{1}{2}\left(-1+\frac{4}{\sqrt{16 m^{4}-16 m^{2}+16}}\right)+\frac{4 m^{2}}{\sqrt{16 m^{4}-16 m^{2}+16}}\right)- \\
& \frac{3}{2 \sqrt{m^{4}-m^{2}+1}} \mathrm{~ns}^{2}\left(\frac{1}{\sqrt[4]{16 m^{4}-16 m^{2}+16}} \xi \mid m\right), \\
& v_{3}(\xi)=\frac{1}{2}\left(-1+\frac{4}{\sqrt{16 m^{4}+224 m^{2}+16}}+\frac{4 m^{2}}{\sqrt{16 m^{4}+224 m^{2}+16}}\right)- \\
& \frac{3 m^{2}}{2 \sqrt{m^{4}+14 m^{2}+1}} \operatorname{sn}^{2}\left(\frac{1}{\sqrt[4]{16 m^{4}+224 m^{2}+16}} \xi \mid m\right)- \\
& \frac{3}{2 \sqrt{m^{4}+14 m^{2}+1}} \mathrm{~ns}^{2}\left(\frac{1}{\sqrt[4]{16 m^{4}+224 m^{2}+16}} \xi \mid m\right), \\
& v_{4}(\xi)=\frac{1}{2}\left(-1-\frac{1}{\sqrt{m^{4}-m^{2}+1}}-\frac{m^{2}}{\sqrt{m^{4}-m^{2}+1}}\right)+ \\
& \frac{6 m^{2}}{\sqrt{16 m^{4}-16 m^{2}+16}} \operatorname{sn}^{2}\left(\frac{i}{\sqrt[4]{16 m^{4}-16 m^{2}+16}} \xi \mid m\right), \\
& v_{5}(\xi)=\frac{1}{2}\left(-1-\frac{1}{\sqrt{m^{4}-m^{2}+1}}-\frac{m^{2}}{\sqrt{m^{4}-m^{2}+1}}\right)+ \\
& \frac{6}{\sqrt{16 m^{4}-16 m^{2}+16}} \mathrm{~ns}^{2}\left(\frac{i}{\sqrt[4]{16 m^{4}-16 m^{2}+16}} \xi \mid m\right), \\
& v_{6}(\xi)=(\xi)=\frac{1}{2}\left(-1-\frac{1}{\sqrt{m^{4}+14 m^{2}+1}}-\frac{m^{2}}{\sqrt{m^{4}+14 m^{2}+1}}\right)+ \\
& \frac{6 m^{2}}{\sqrt{16 m^{4}+224 m^{2}+16}} \operatorname{sn}^{2}\left(\frac{i}{\sqrt[4]{16 m^{4}+224 m^{2}+16}} \xi \mid m\right)+ \\
& \frac{6}{\sqrt{16 m^{4}+224 m^{2}+16}} \mathrm{~ns}^{2}\left(\frac{i}{\sqrt[4]{16 m^{4}+224 m^{2}+16}} \xi \mid m\right) \text {. }
\end{aligned}
$$


Letting $m \longrightarrow 1$, we obtain hyperbolic solutions:

$$
\begin{aligned}
& v_{1}^{*}(\xi)=\frac{1}{2}-\frac{3}{2} \tanh ^{2}\left(\frac{\xi}{2}\right), \\
& v_{2}^{*}(\xi)=\frac{1}{2}-\frac{3}{2} \operatorname{coth}^{2}\left(\frac{\xi}{2}\right), \\
& v_{3}^{*}(\xi)=-\frac{1}{4}-\frac{3}{8} \operatorname{coth}^{2}\left(\frac{\xi}{4}\right)-\frac{3}{8} \tanh ^{2}\left(\frac{\xi}{4}\right) .
\end{aligned}
$$

Also, we have derived trigonometric solutions when $m \longrightarrow 0$ :

$$
\begin{aligned}
& v_{4}^{*}(\xi)=-\frac{3}{2} \sec ^{2}\left(\frac{\xi}{2}\right), \\
& v_{5}^{*}(\xi)=-\frac{3}{2} \csc ^{2}\left(\frac{\xi}{2}\right) .
\end{aligned}
$$

\section{Conclusion}

In this paper, we have found solutions for BBM equation given in (2) by both the tanh-coth method and the sn-ns method. Our purpose is to compare the solutions observed by these two methods. If we check the solutions, it can be seen that same hyperbolic solutions are obtained by these two methods. Even if we have the same solutions for equation (2) in terms of the hyperbolic functions, the sn-ns method usually introduces more solutions than the tanh-coth method. And also, the solutions observed by the sn-ns method always contains the solutions found by the tanh-coth method, since the tanh-coth method is a specific case of the sn-ns method. Moreover, the sn-ns method gives the trigonometric and elliptic function solutions in addition to the hyperbolic ones. Thus, it can be said that the sn-ns method is more powerful for getting solutions of the most NPLDEs.

\section{REFERENCES}

[1] E. Fan and Y.C. Hon, Generalized tanh method extended to special types of nonlinear equations, Z. Naturforsch, Vol. 57 (2002), pp. 692-700.

[2] A.-M. Wazwaz, The extended tanh method for new solitons solutions for many forms of the fifth order KdV equations, Appl. Math. Comput., Vol. 184 (2007), pp. 1002 1014.

[3] A.-M. Wazwaz, New travelling wave solutions of different physical structures to generalized BBM equation, Phys. Lett. A., Vol. 355 (2006), pp. 358-362.

[4] A.-M. Wazwaz, M.A. Helal, Non linear variants of the BBM equation with compact and noncompact physical structures, Chaos, Solitons and Fractals, Vol. 26 (2005), pp. $767-776$.

[5] J.S. Yadong, Explicit and exact special solutions for BBM-like B $(m, n)$ equations with fully nonlinear dispersion, Chaos, Solitons and Fractals, Vol. 25 (2005), pp. 1083-1091.

[6] C.T. Yan, A simple transformation for nonlinear waves, Phys Lett A, Vol. 224 (1996), pp. $77-84$. 
[7] J.-H. He and L.-N. Zhang, Generalized solitary solution and compacton-like solution of the Jaulent-Miodek equations using the Exp-function method, Phys. Lett. A., Vol. 372 (2008), pp. 1044-1047.

[8] R. Conte and M. Musette, Link between solitary waves and projective Riccati equations, J. Phys. A-Math. Gen., 25 (1992) 5609-5623.

[9] Z. Yan, The Riccati equation with variable coefficients expansion algorithm to find more exact solutions of non linear differential equations, Comput. Phys. Comm., Vol. 152 (2003), pp. 1-8.

[10] M. Wang, X. Li, J. Zahng, The $\left(G^{\prime} / G\right)$-expansion method and travelling wave solutions of nonlinear evolution equations in mathematical physics, Phys. Lett. A., Vol. 372 (2008), pp. 417-423.

[11] H.S. Alvaro, Solving nonlinear partial differential equations by the sn-ns method, Abstr. Appl. Analysis., Vol. 25 (2012), pp. 1-25.

[12] T.B. Benjamin, J.L. Bona, J.J. Mahony, Model equations for long waves in nonlinear dispersive systems, Philos. Trans. R. Soc. London, Ser A, Vol. 272 (1972), pp. 47-48.

[13] D.J. Korteweg, G. De Vries, On the change of long waves advancing in a rectangular canal and a new type of long stationary wave, Phil. Mag., Vol. 39 (1835), pp. 422-443.

[14] M.A. Raupp, Galerkin methods applied to the Benjamin-Bona-Mahony equation, Bull. Braz. Math. Soc., Vol. 6 (1975), pp. 65-77.

[15] L. Wahlbin, Error estimates for a Galerkin method for a class of model equations for long waves, Numer. Math., Vol. 23 (1975), pp. 289-303.

[16] R.E. Ewing, Time-stepping Galerkin methods for nonlinear Sobolev partial differential equation, SIAM J. Numer. Anal., Vol. 15 (1978), pp. 1125-1150.

[17] Arnold, D.N, J. Jr, V, Thomee, Superconvergence of finite element approximation to the solution of a Sobolev equation in a single space variable, Math. Comput., Vol. 27 (1981), pp. 737-743.

\author{
HAMi GüNDOĞDU \\ Department of Mathematics \\ Faculty of Sciences and Art \\ SAKARYA UNIVERSITY \\ SAKARYA, 54187 AdAPAZARI \\ TURKEY \\ E-mail address: hamigundogdu@sakarya.edu.tr
}

\section{ÖMER FARUK GözüKIZIL}

Department of Mathematics

Faculty of ScIEnces ANd ART

SAKARYA UNIVERSITY

SAKARYA, 54187 AdAPAZARI

TURKEY

E-mail address: farukg@sakarya.edu.tr 